\title{
Changes in evidence for studies assessing interventions for COVID-19 reported in preprints: meta-research study
}

Theodora Oikonomidi ${ }^{1,2,3^{*}}$ (D), Isabelle Boutron ${ }^{1,2,3}$, Olivier Pierre ${ }^{1,2,3}$, Guillaume Cabanac ${ }^{4} \mathbb{D}$, Philippe Ravaud ${ }^{1,2,3,5}$ and the COVID-19 NMA Consortium

\begin{abstract}
Background: The increasing use of preprints to disseminate evidence on the effect of interventions for the coronavirus disease 2019 (COVID-19) can lead to multiple evidence sources for a single study, which may differ in the reported evidence. We aim to describe the proportion of evidence on the effect of interventions for COVID-19 from preprints and journal articles and map changes in evidence between and within different sources reporting on the same study.

Methods: Meta-research study. We screened the Cochrane living systematic review and network meta-analysis (COVID-NMA) database to identify all preprints and journal articles on all studies assessing interventions for COVID19 published up to 15 August 2020. We compared all evidence sources (i.e., preprint and associated journal article) and the first and latest versions of preprints for each study to identify changes in two evidence components: study results (e.g., numeric change in hazard ratio, odds ratio, event rate, or change in $p$ value $>$ or $<0.05$ in any outcome) and abstract conclusions (classified as positive, negative or neutral regarding the intervention effect, and as reporting uncertainty in the findings or not). Changes in study results were further classified as important changes if they (1) represented a change in any effect estimate by $\geq 10 \%$ and/or (2) led to a change in the $p$ value crossing the threshold of 0.05 .

Results: We identified 556 studies. In total, 338 (61\%) had been reported in a preprint: 66 (20\%) of these had an associated journal article (median time to publication 76 days [interquartile range (IQR) 55-106]) and 91 (27\%) had $>1$ preprint version. A total of 139 studies (25\% of the overall sample) were reported in multiple evidence sources or versions of the same source: for $63(45 \%)$, there was a change in at least one evidence component between or within sources (42 [30\%] had a change in study results, and in 29 [21\%] the change was classified as important; 33 [24\%] had a change in the abstract conclusion). For studies with both a preprint and an article, a median of 29\% (IQR 14-50) of total citations were attributed to the preprint instead of the article.
\end{abstract}

(Continued on next page)

\footnotetext{
* Correspondence: theodora.oikonomidi@inserm.fr

'Université de Paris, UMR 1153 CRESS Inserm, 75004 Paris, France

${ }^{2}$ Clinical Epidemiology Unit, Hôtel-Dieu Hospital, Assistance

Publique-Hôpitaux de Paris, (AP-HP), 75004 Paris, France

Full list of author information is available at the end of the article
}

(C) The Author(s). 2020 Open Access This article is licensed under a Creative Commons Attribution 4.0 International License, which permits use, sharing, adaptation, distribution and reproduction in any medium or format, as long as you give appropriate credit to the original author(s) and the source, provide a link to the Creative Commons licence, and indicate if changes were made. The images or other third party material in this article are included in the article's Creative Commons licence, unless indicated otherwise in a credit line to the material. If material is not included in the article's Creative Commons licence and your intended use is not permitted by statutory regulation or exceeds the permitted use, you will need to obtain permission directly from the copyright holder. To view a copy of this licence, visit http://creativecommons.org/licenses/by/4.0/. The Creative Commons Public Domain Dedication waiver (http://creativecommons.org/publicdomain/zero/1.0/) applies to the data made available in this article, unless otherwise stated in a credit line to the data. 
(Continued from previous page)

Conclusions: Results on the effect of interventions for COVID-19 are often reported in multiple evidence sources or source versions for a single study. Evidence is not stable between and within evidence sources. Real-time linkage of all sources per study could help to keep systematic reviews up-to-date.

Keywords: Preprint, Meta-research, COVID-19, Coronavirus

\section{Background}

The coronavirus disease 2019 (COVID-19) pandemic has created a need for rapid performance and dissemination of systematic reviews on the effectiveness of preventive, therapeutic, and post-acute care interventions to guide clinical practice, policy-making, and guideline development [1]. Researchers compiling systematic reviews are faced with the challenge of synthesizing large amounts of evidence disseminated rapidly, principally through two channels of communication: preprint servers and journals [2].

Systematic reviews traditionally rely on peer-reviewed journal articles, which represent the final form of a study report, and may only undergo changes through retraction or publication of a correction. However, in the context of COVID-19, many researchers have chosen to disseminate their findings by using preprints (i.e., nonpeer-reviewed manuscripts, accessible on open archives). Preprints offer several benefits. First, they are published rapidly. For example, publication on medRxiv, the preprint server for the health sciences, takes only 5 days [3]. In comparison, publication in a peer-reviewed biomedical journal can take several months. Second, preprints can easily be updated as additional data are obtained and analyzed, by uploading a new version of the manuscript on the preprint platform. Ultimately, preprint servers help disseminate evidence at the rapid pace imposed by the COVID-19 pandemic.

However, the use of preprints can lead to multiple evidence sources (i.e., the preprint and the associated journal article) as well as multiple versions of the same evidence source (i.e., the first and updated preprint versions) being available for a single study. Important information, such as outcome effect sizes, could undergo changes between and within these sources as evidence for a single study evolves over time. Multiplicity of incongruent evidence sources can mislead systematic reviewers and other research end-users.

In this study, we aimed to (1) describe the proportion of evidence on the effect of interventions for COVID-19 from preprints and journal articles, (2) map changes in evidence for the same study between different sources (i.e., preprint and journal article) and between different versions of the same source (i.e., preprint versions), and (3) describe how results from each source are disseminated by using the citation count, Altmetric Attention Score, and PubPeer comments.

\section{Methods}

This meta-research study is ancillary to the Cochrane living systematic review and network meta-analysis (NMA) on COVID-19 (hereafter COVID-NMA) (https://covid-nma.com/, PROSPERO CRD42020182600) [4]. COVID-NMA is a living systematic review, in which all available evidence related to COVID-19 is continuously collected, critically appraised, and synthesized using pairwise comparison and NMA methods. The protocol for the present study can be provided upon request by the corresponding author.

Our sample comprises all studies assessing preventive, therapeutic, or post-acute care interventions for COVID-19 that were published as preprints or journal articles up to 15 August 2020.

\section{Data sources}

To identify eligible studies, we screened the COVIDNMA database, which provides an exhaustive archive of COVID-19 studies assessing the effect of interventions. As part of the COVID-NMA project, medRxiv, and PubMed are searched daily, independently by two reviewers, to identify eligible studies. Secondary sources, such as the Living OVerview of Evidence (L.OVE) database, are searched as a quality control. The search strategy is in Additional file 1: Methods S1.

\section{Eligibility criteria and study selection}

We included all preprints and journal articles indexed in the COVID-NMA database, from inception to 15 August 2020 , with the following study designs: randomized controlled trials (RCTs), observational studies (i.e., case series, case-control, cohort, interrupted time series, studies modeling the effects of population-level interventions). We excluded case series with $<5$ participants, systematic reviews, prognosis and diagnosis studies, and modeling studies using simulation methods. We included reports on preventive, therapeutic, and postacute care interventions, for healthy individuals, patients with COVID-19, or recovered individuals, respectively. Because the scope of the COVID-NMA is already very broad, studies assessing Traditional Chinese Medicine and other types of Traditional, Complementary and Alternative Medicine (TM/CAM) interventions were excluded [4]. Studies not performed with humans were also excluded. 
Two reviewers (T.O. and O.P.) independently screened all records. Disagreements were resolved by consensus or by consultation with a senior reviewer (I.B.).

\section{Linking all evidence sources for each study}

We sought to link preprints and journal articles reporting results from the same study. For this purpose, we used a four-step approach:

1. We performed a systematic search of preprints and published articles as part of the COVID-NMA (as described above). We used the same eligibility criteria for preprints and articles. One author (T.O.) downloaded these data in Excel format and used three ways to identify potential preprint-article pairs: (a) by using the Excel search function to identify articles for which the name of the article author matched that of the first author of an included preprint, and comparing the titles of potential matches to verify that they reported the same study; (b) by extracting the intervention type assessed in each preprint and article and comparing the titles of preprints and articles that assessed the same intervention to identify pairs that reported the same study; and (c) by comparing the trial registration number for preprints and articles reporting RCTs.

2. We performed additional searches of the Dimensions academic search engine (https://app.dimensions.ai). We used the name of the first author of each preprint to identify articles by the same author in any authorship position. We did this by downloading the Dimensions dataset in Excel format, filtering entries that were not journal articles, and using the search function to search for the name of the preprint's first author in the column containing all article author names. When a potential preprint-article pair was identified in this manner, we compared the titles and the names of the remaining authors to decide whether the two sources reported findings from the same study.

3. One author (G.C.) designed an algorithm that relies on preprint metadata obtained via the Crossref API (https://github.com/CrossRef/rest-api-doc) (e.g., author ORCIDs, author names, sequence of coauthors listed in the bylines, title words, preprint/ publication timelines) to identify journal articles reporting on the same study as the included preprints. All links identified by the algorithm were then reviewed by one author (T.O.) to remove false-positives. Furthermore, the accuracy of the algorithm was validated by comparing its results for 740 preprint-journal article pairs, against all links established by the medRxiv platform as of 14 July 2020. The algorithm correctly linked $99.73 \%$ of the
740 pairs, with only 2 false-negatives. Detailed results on the accuracy of the algorithm will be reported in a validation study [Cabanac et al., unpublished data].

4. Finally, we contacted the corresponding author of each preprint that was classified as unpublished in the above two steps via email (see Additional file 1: Methods S2 for a template) to ask whether the preprint had been accepted for journal publication. For preprints that had not been accepted, we asked whether they had been submitted to a journal, or if the authors intended to submit them in the future. We sent a reminder email to authors who did not respond to our first email. We received 123 responses from the 272 authors contacted (45\% response rate). No new preprint-journal article links were identified in this step.

In this report, we include preprint-journal article links identified up to 2 September 2020.

\section{Data extraction}

We extracted the following general characteristics of studies: study design, intervention, country of affiliation for the corresponding author, and data sharing (i.e., whether the authors were willing to share the dataset used in the study or not, from the data sharing statement).

\section{Characteristics of evidence sources}

We classified each source as a preprint or journal article. For journal articles, we manually extracted whether the journal had issued corrections or a retraction notice by searching the journal website and Retraction Watch (https://retractionwatch.com/retracted-coronavirus-covid-19-papers/). We used an algorithm to automatically extract the number of versions on the preprint server for each preprint and the date of online publication for each preprint and journal article.

\section{Changes between and within evidence sources}

We identified all studies in our sample for which there was more than one evidence source (i.e., a preprint and a journal article) or more than one version of the same evidence source (i.e., $>1$ preprint version).

First, we sought to compare important evidence components between the following evidence sources:

- First preprint version versus journal article

- Latest preprint version versus journal article (if $>1$ preprint versions were available)

Second, we sought to compare evidence components within evidence sources (the first preprint version versus the latest preprint version, if $>1$ versions were available). 
To perform these comparisons, we downloaded all abovementioned sources for each study in portable document format (PDF). We entered each pair of files in PDF Converter (Enterprise 8). This software allows users to compare documents by automatically detecting and highlighting changes in text (words added or deleted between versions). We reviewed the highlighted text to identify changes in the following evidence components, which may affect systematic reviewers' appraisal of the effect of the intervention assessed in the study:

1) Change in any study result. We searched for numeric changes in at least one of the following effect-size metrics: hazard ratio, odds ratio, relative risk, event rate, correlation or regression coefficient (for country-level studies), or in the statistical significance (i.e., $p$ value changed from $>$ to $<0.05$, or vice versa), for any outcome. To characterize the magnitude of change in results, we considered the change to be important if (1) it represented an increase or decrease by $\geq 10 \%$ of the initial value in any effect estimate and/or (2) it led to a change in the $p$ value crossing the threshold of 0.05 . For evidence source pairs that had a change in results, we additionally extracted whether the sample size had changed among or between evidence sources. The sample size was defined as the number of individuals enrolled in the study or the number of countries/regions analyzed (for population-level studies of policy interventions).

2) Change in the study conclusion reported in the abstract. First, we assessed the conclusion to determine two aspects:

- If the abstract conclusion was positive, neutral, or negative regarding the effect of the intervention (i.e., whether the authors focused on improvement in any efficacy outcome or reduction in harms; versus lack of impact on any outcome; versus focus on deterioration of efficacy or safety outcomes).

- If the abstract conclusion reported uncertainty in the findings (i.e., whether the authors emphasized the need for additional studies to confirm the findings and/or used mild phrasing such as "might be effective" versus strong phrasing such as "These results prove the efficacy of the intervention").

We considered any change in conclusion among positive, neutral, or negative or change between reporting versus not reporting uncertainty to constitute change in the conclusion.

\section{Dissemination of evidence sources}

To describe the dissemination of the different evidence sources, we used an algorithm designed by one of the authors (G.C.) to automatically extract the following usage data for each preprint-journal article pair: citation count, Altmetric Attention Score (extracted from the Dimensions database) [5], and the number of PubPeer comments (extracted from the PubPeer Application Programming Interface $[\mathrm{API}])$. These metrics were selected because they represent evidence dissemination by different end-user groups. Citations reflect use of an evidence source in academic communications. The Altmetric Attention Score tracks the occurrences of a source mentioned in the media and online bibliographic reference managers [6]. Finally, PubPeer data reflect attention by researchers in the form of crowdsourced peer review. Commenters can write comments or ask for clarifications about the study and the study authors can post a reply [7]. Usage data were last updated on 21 October 2020.

\section{COVID-NMA subgroup}

We sought to identify changes in evidence in a subgroup of studies currently being used for quantitative evidence synthesis in the COVID-NMA. These are RCTs and non-randomized studies that are interrupted time series or non-randomized studies using causal inference analysis or multivariable regression adjustment, with $\geq 150$ incident users.

In addition to the study results and conclusion, we searched for changes in the following methodologic components that could affect the appraisal of risk of bias $[8,9]$ : blinding of participants, clinicians, or outcome assessors; amount and handling of missing data; randomization process and allocation concealment (in RCTs); inclusion of participants in the study or the analysis; and statistical method used to adjust for confounders and confounders adjusted for (in nonrandomized studies).

One reviewer (T.O.) extracted data for all included studies by using a structured, piloted form (see Additional file 1: Methods S3). A second reviewer (O.P.) independently extracted data from all sources for $20 \%$ of the studies for all variables except for assessment of the conclusion, which was duplicated in full. Disagreements were resolved by consensus or by consulting a senior reviewer (I.B.). The agreement between reviewers was > $80 \%$ for all variables.

\section{Data analysis}

We used descriptive statistics to describe study characteristics and changes in evidence. We summarized data usage (e.g., citation count) as median and interquartile range (IQR). Because our sample included evidence 


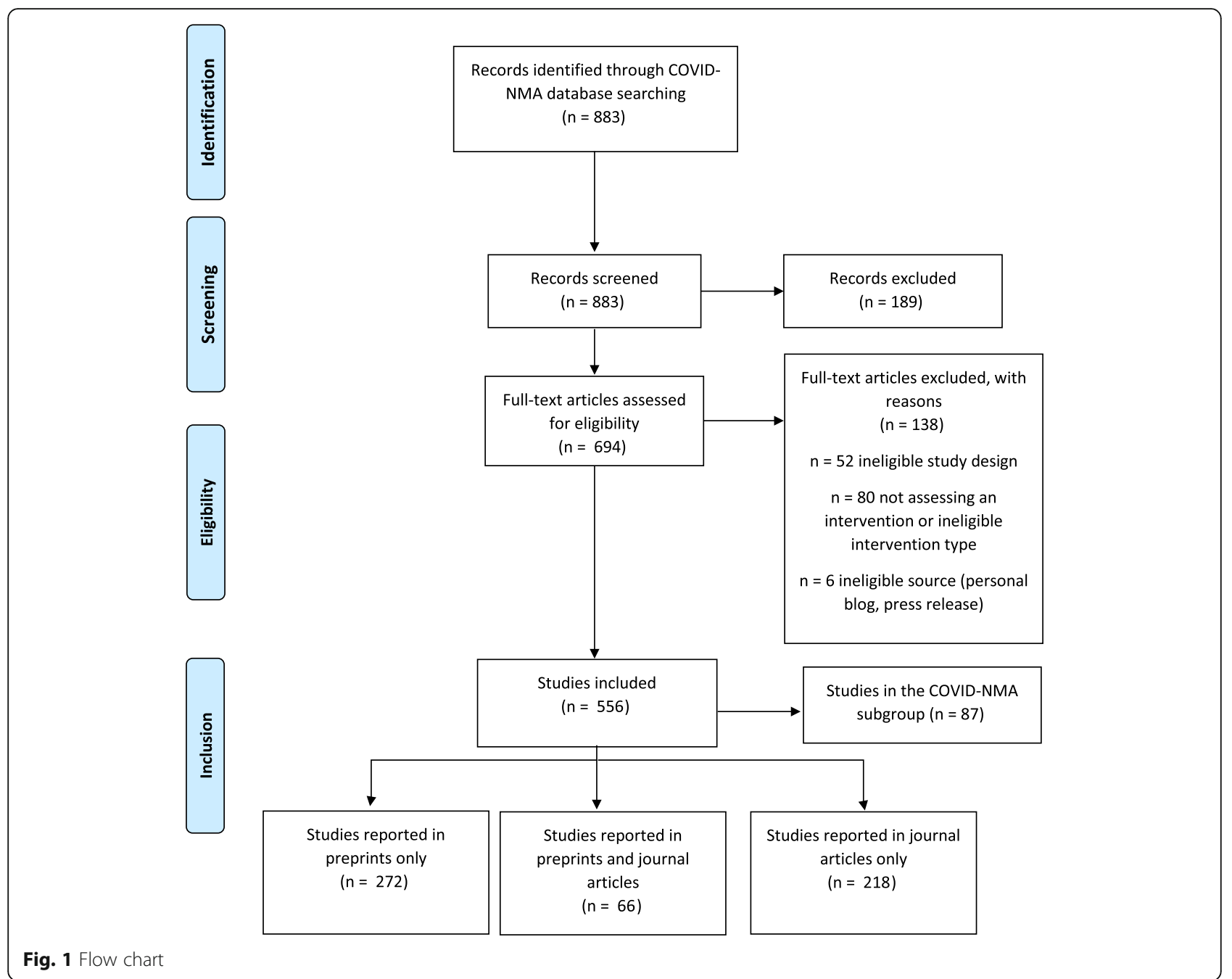

sources published over an 8-month period, we accounted for the difference in exposure time by dividing each usage metric by the number of days since the date of first online publication.

\section{Results}

We identified 556 studies assessing interventions for COVID-19 (Fig. 1). The most commonly assessed interventions were social distancing/lockdown policy measures $(n=90,17 \%)$ and antimalarial agents $(n=76,14 \%)$ (Table 1$)$. In half of the studies, the country of affiliation for the corresponding author was China $(n=144,26 \%)$ or the USA ( $n=135,24 \%)$.

\section{Characteristics of evidence sources in the overall sample} Overall, 61\% $(n=338)$ of all studies had been reported in a preprint (Table 1$)$. The remaining 39\% $(n=218)$ were directly published as journal articles. Among the 338 studies reported as preprints, 66 (20\%) had then been published as journal articles. The median delay from publication of the first preprint version to publication of the associated journal article was 76 days (IQR 55-106) (Fig. 2).

We obtained additional information about the status of the 272 unpublished preprints by contacting the authors. We received responses for 123 preprints: 2 had been accepted for publication in a journal, 107 (32\%) had been submitted for publication, and 9 (3\%) will be submitted. A small proportion of authors $(n=5,1 \%)$ did not intend to submit the preprint to a journal (Table 1).

Regarding the update of evidence over time, for $27 \%$ ( $n=91$ ) of preprints, $>1$ version was uploaded to the preprint server (median number of versions per preprint: 1 [IQR $1-2$, range 1 to 6$]$ ), and $2 \%$ of journal articles had been updated ( $n=1$ retracted, $n=4$ had a published correction).

\section{Changes between and within evidence sources in the overall sample}

We found multiple sources of information for 139 (25\%) studies: 66 had a preprint and a journal article and 91 
Table 1 Characteristics of 556 studies assessing interventions for COVID-19 prevention, treatment or rehabilitation

\begin{tabular}{ll}
\hline Characteristics & $\boldsymbol{n}=\mathbf{5 5 6}$ \\
\hline Intervention (\%) $^{\mathbf{a}}$ & \\
Social distancing, lockdown, or travel restrictions & $90(17)$ \\
Antimalarials & $76(14)$ \\
Monoclonal antibodies & $52(10)$ \\
Corticosteroids & $37(7)$ \\
Other & $288(53)$ \\
Country of affiliation for the corresponding author (\%) & \\
China & $144(26)$ \\
USA & $135(24)$ \\
Italy & $39(7)$ \\
France & $32(6)$ \\
UK & $26(5)$ \\
Other & $180(32)$
\end{tabular}

Data sharing (\%)

Data will be shared upon request to the authors

Publicly available dataset

No data sharing

Unclear or unreported

Has a peer-reviewed evidence source (\%)

Evidence source (\%)

Preprint

Journal article

Preprint plus journal article

Status of studies initially published as preprints

Published in a journal (\%)

$n=338$

Accepted for publication (\%)

$66(20)$

$2(0)$

Unpublished, submitted to a journal

107 (32)

Unpublished, will be submitted to a journal

9 (3)

Unpublished, will not be submitted to a journal

Unpublished, unknown status (no response from authors)

\begin{abstract}
${ }^{a}$ May not add up to $100 \%$ because of rounding
bother interventions include health care organization, respiratory support, combination antivirals, antiretrovirals, angiotensin-converting enzyme inhibitor/angiotensin II receptor blocker, convalescent plasma, personal protective equipment, non-specific antiviral, broad-spectrum antiviral, anticoagulant, gas inhalation, advanced therapy medicinal products, organ support, population testing, antibiotics, non-steroidal anti-inflammatory drugs, respiratory stimulants, antiparasitic, immunosuppressants, kinase inhibitors, radiation, rehabilitation calcium channel blockers, and vaccination 'Other countries include Abu Dhabi, Albania, Australia, Belgium, Brazil, Brunei, Canada, Chile, Cuba, Denmark, Germany, Greece, Hong-Kong, India, Iran, Ireland, Israel, Japan, Jordan, Malaysia, Mexico, New Zealand, Norway, Portugal, Qatar, Saudi Arabia, Singapore, Slovakia, Spain, South Africa, South Korea, Sri Lanka, Switzerland, Thailand, The Netherlands, Tunisia, Turkey, Ukraine, and Vietnam. All $<4 \%$ of total sample
\end{abstract}

had $>1$ preprint version (18 studies had both a journal article and $>1$ preprint version). For $45 \%(n=63)$ of studies, we identified changes in at least one evidence component between or within evidence sources for the same study (Table 2). In total, 30\% $(n=42)$ of studies had changes in study results and $24 \%(n=33)$ had changes in the abstract conclusion. Regarding important changes in results (i.e., changes representing an increase or decrease by $\geq 10 \%$ of the initial effect estimate and/or leading to a change in $p$ value at the 0.05 threshold), $21 \%(n=29)$ of studies had important changes in study results (Additional file 1: Table S1). Among the total 54 changes in results observed between and within all evidence pairs, 18 (33\%) evidence pairs also had a change in sample size.

Of all changes in conclusion $(n=40$ changes; some studies had changes both between and within evidence sources), the most common change was from positive without reporting uncertainty, to positive with reporting of uncertainty $(n=12)$. In terms of changes among positive, neutral, and negative, the most common changes were from neutral to positive $(n=6)$ (Fig. 3).

\section{Characteristics of evidence sources in the COVID-NMA subgroup}

There were 87 studies in the COVID-NMA subgroup, which consists of RCTs and observational studies included in the Cochrane living systematic review and is available online to support decision-makers. Overall, $64 \%(n=56)$ had been reported in a preprint, $25 \%(n=$ 14) had then been published as journal articles, and $36 \%$ $(n=31)$ were directly published as journal articles. The median delay from the publication of the first preprint version to publication of the associated journal article was 74 days (IQR $36-97$ ). For $30 \%$ of preprints, > 1 version was uploaded to the preprint server (median number of versions per preprint: 1 [IQR $1-2$, range 1 to 4 ]). One journal article had been retracted and one had a published correction.

\section{Changes between and within evidence sources in the COVID-NMA subgroup}

In total, 25 studies of the COVID-NMA subgroup had multiple evidence sources or multiple versions of the same source (Table 2). We identified changes in any evidence component, between any evidence source, for 15 (60\%) studies (Table 2). Changes were identified most frequently in study results $(40 \%, n=10$, with any change in results, and $20 \%, n=5$, with an important change in results). Five studies (20\%) had changes in methodologic components that may affect risk of bias assessment and $8(32 \%)$ had changes in the abstract conclusion.

Finally, we identified the following changes that may affect risk of bias assessment: (1) addition of information about the propensity score model of non-randomized studies, such as additional confounders entered in the model, and specification of the propensity score being time-dependent $(n=2) ; \quad(2)$ reporting of additional 


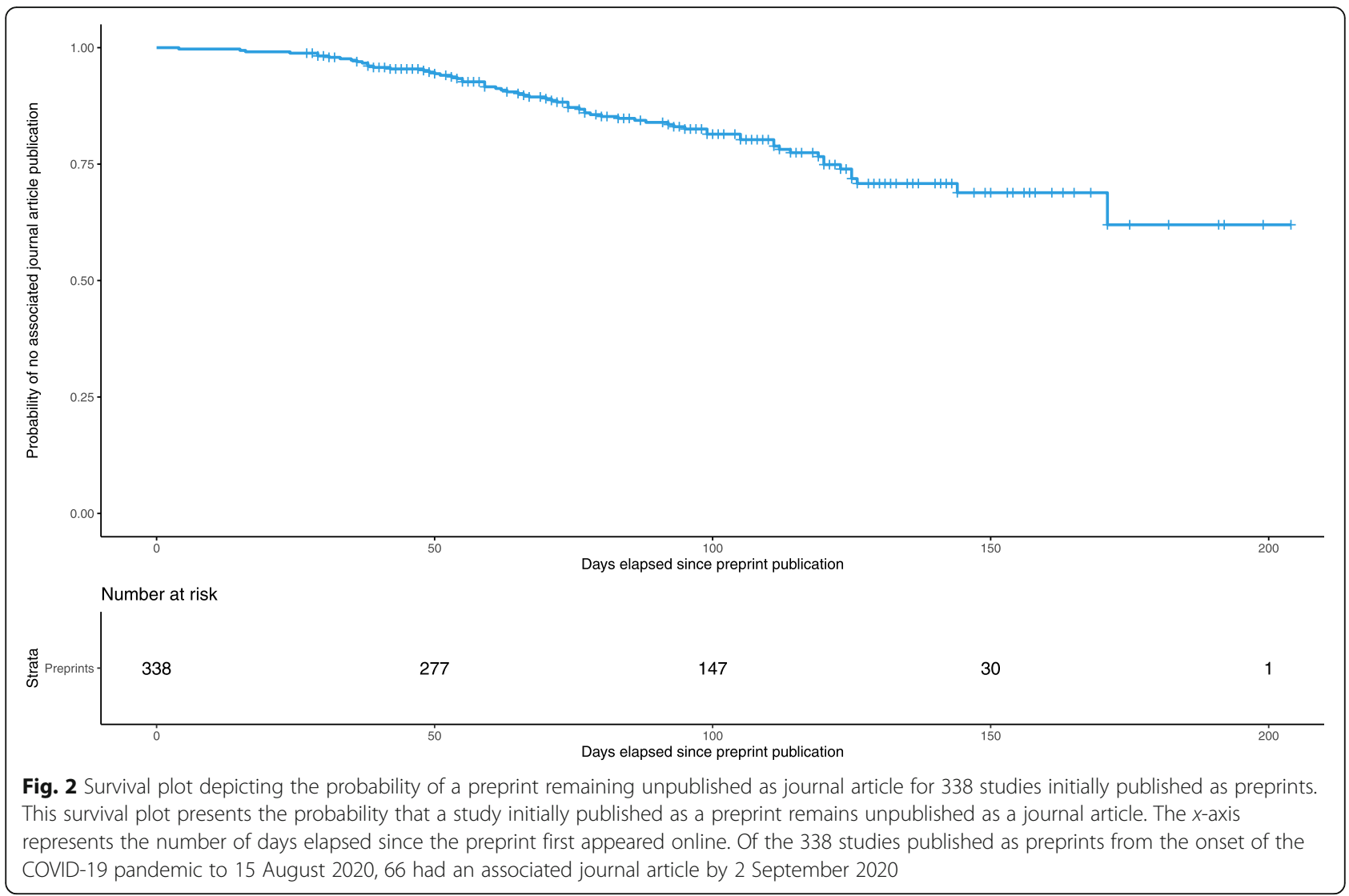

information about the conduct of the study that indicates prevalent user bias $(n=1)$; and (3) addition or removal of information about allocation concealment in $\operatorname{RCTs}(n=2)$.

\section{Dissemination of evidence sources}

Usage data for preprint-journal article pairs are presented in Additional file 1: Table S2 and Additional file 1: Figure S1. More preprints than articles received 0 citations $(n=10$ of 65 [usage data could not be retrieved for 1 preprint], $15 \%$, and $n=3$ of $66,5 \%$, respectively). Of the total citations for each study, a median of $29 \%$ (IQR 14-50) was attributed to preprints.

A similar proportion of preprints and articles had an Altmetric Attention score of $0(n=4,6 \%$ for both preprints and articles). Furthermore, we examined the breakdown of the Altmetric Score for the 66 preprint-journal article pairs. Specifically, we examined their score in the Policy documents domain, which reflects the citations a report has received in policy documents. Of the 66 preprint-journal article pairs, 9 preprints $(14 \%)$ and 10 articles (15\%) had a score $>0$ in the Policy documents domain (median score for preprints 0 , IQR 0-0; median score for articles 0 , IQR 0-0).
Finally, 92\% of preprints and 95\% of journal articles had 0 PubPeer comments. For both the preprints and journal articles with PubPeer comments, the median number of comments was 0 (IQR 0-0).

\section{Discussion}

This meta-research study showed that results for most studies assessing the effect of interventions for COVID19 were initially disseminated in preprints, and the delay in publication of these results in journal articles takes 2 months. One-third of studies disseminated as preprints had at least one update since they first appeared online, with the addition of either more preprint versions or associated journal articles. Nearly half of these studies had changes in at least one important evidence component between or within evidence sources.

Preprints make a vital contribution to the body of evidence on the effect of interventions for COVID-19. The delay between preprint publication and journal article publication is 76 days (IQR 55-106); hence, for several months, preprints constitute the main source of evidence for a study. However, preprints constitute "living evidence" that is not stable. This flexibility allows researchers to keep up with evolving datasets (e.g., in studies exploring the association between policy interventions and mortality rates) and disseminate 
Table 2 Change in evidence components, between and within evidence sources, in the overall sample and in a subgroup of randomized controlled trials and observational studies usable for quantitative evidence synthesis ${ }^{\mathrm{a}}$

\begin{tabular}{|c|c|c|c|c|c|c|c|c|}
\hline & Overall sample $(n=1$ & $139)^{b}$ & & & COVID-NMA subg & up $(n=25$ & & \\
\hline & $\begin{array}{l}\text { Change between or } \\
\text { within at least one } \\
\text { evidence source } \\
\text { pair }(n=139)\end{array}$ & $\begin{array}{l}\text { First to } \\
\text { latest } \\
\text { preprint } \\
\text { version } \\
(n=91)\end{array}$ & $\begin{array}{l}\text { First } \\
\text { preprint } \\
\text { version to } \\
\text { journal } \\
\text { article ( } n= \\
66 \text { ) }\end{array}$ & $\begin{array}{l}\text { Latest } \\
\text { preprint } \\
\text { version to } \\
\text { journal } \\
\text { article ( } n= \\
\text { 18) }\end{array}$ & $\begin{array}{l}\text { Change between } \\
\text { or within at least } \\
\text { one evidence } \\
\text { source pair ( } n= \\
25 \text { ) }\end{array}$ & $\begin{array}{l}\text { First to } \\
\text { latest } \\
\text { preprint } \\
\text { version } \\
(n=18)\end{array}$ & $\begin{array}{l}\text { First } \\
\text { preprint } \\
\text { version to } \\
\text { journal } \\
\text { article }(n= \\
15)\end{array}$ & $\begin{array}{l}\text { Latest } \\
\text { preprint } \\
\text { version to } \\
\text { journal } \\
\text { article }(n=7)\end{array}$ \\
\hline $\begin{array}{l}\text { Change in at } \\
\text { least } 1 \\
\text { evidence } \\
\text { component }\end{array}$ & $63(45)$ & $35(38)$ & $36(55)$ & $8(44)$ & $15(60)$ & $8(44)$ & $12(86)$ & $3(43)$ \\
\hline $\begin{array}{l}\text { Change in } \\
\text { study results }\end{array}$ & $42(30)$ & $23(25)$ & $25(38)$ & $6(33)$ & $10(40)$ & $5(28)$ & $9(64)$ & $3(43)$ \\
\hline $\begin{array}{l}\text { Change in risk } \\
\text { of bias } \\
\text { assessment }\end{array}$ & - & - & - & - & $5(20)$ & $1(6)$ & $4(27)$ & $1(14)$ \\
\hline $\begin{array}{l}\text { Change in } \\
\text { abstract } \\
\text { conclusion }\end{array}$ & $33(24)$ & $18(20)$ & $19(29)$ & $3(17)$ & $8(32)$ & $5(28)$ & $5(36)$ & $1(14)$ \\
\hline
\end{tabular}

Includes studies used in quantitative evidence synthesis and GRADE development in the COVID-NMA project (randomized controlled trials; interrupted timeseries, non-randomized studies using causal inference analysis or multivariable regression adjustment, including at least 150 incident users), with multiple evidence sources or evidence source versions. For more information see: https://covid-nma.com/emulated/

${ }^{\mathrm{b}}$ Includes the studies in the COVID-NMA subgroup

findings from interim analyses. Therefore, it is possible to observe changes in the reported evidence among sources for the same study, even though both sources were accurate at the time of redaction. For example, the first version of a preprint may accurately report the findings of an interim analysis of an RCT, before participant enrollment has been completed. The final version of the preprint, reporting the analysis of the complete dataset could have a different sample size and different results. The flexibility of preprints also allows authors to correct potential errors in the report following crowd-sourced peer review (e.g., in the form of PubPeer comments). One consequence of this flexibility is that evidence changes can lead to misleading results of systematic reviews. Systematic reviewers need to be aware of updates in the evidence reported in preprints in order to update the review accordingly. One such example is the ELACOI RCT [10]. The first preprint version of the ELACOI RCT reported findings for 44 participants, with $24 \%$ of those receiving lopinavir/ritonavir experiencing adverse events [10], but the final version of the preprint, published on medRxiv 3 weeks later, reported findings for 86 participants, with $35 \%$ of those receiving lopinavir/ritonavir experiencing adverse events. The authors did not specify that they were reporting preliminary results in the first preprint version.

To our knowledge, only one other study assessed changes in preprint-article pairs of studies of COVID19 of any design [11]. The authors found changes that strengthened or softened the data and abstract conclusions in $27 \%$ of a randomly selected sample of COVID-
19 preprint-article pairs. Our results are in agreement, although they are not directly comparable, because we focused on studies assessing interventions.

\section{Meaning of the study}

Our findings point to a pressing need for systematic reviewers to monitor the literature for updates so as to use the latest evidence source for each study. This task is near impossible to perform manually (e.g., 6480 preprints were hosted on medRxiv on 1 September 2020) [12]. Despite efforts by preprint servers to alert users when a preprint is published in a journal, these may not be optimal during the pandemic. For example, of the 61 medRxiv preprint-journal article pairs we identified, medRxiv displayed the linkage for only 26 on 2 September 2020.

The large-scale use of preprints is a novel development for the biomedical sciences [13], and new mechanisms to manage this evidence dissemination track need to be invented. The algorithm designed for the present study offers such a mechanism by detecting the appearance of new evidence sources in real-time and summarizing this information in a web interface [14].

\section{Strengths and weaknesses of the study}

This is the first study to assess changes between evidence sources for studies evaluating interventions for COVID-19. First, we used a large database to capture all relevant evidence sources published during the first semester of the pandemic. Second, we assessed changes in important evidence components that may affect the 


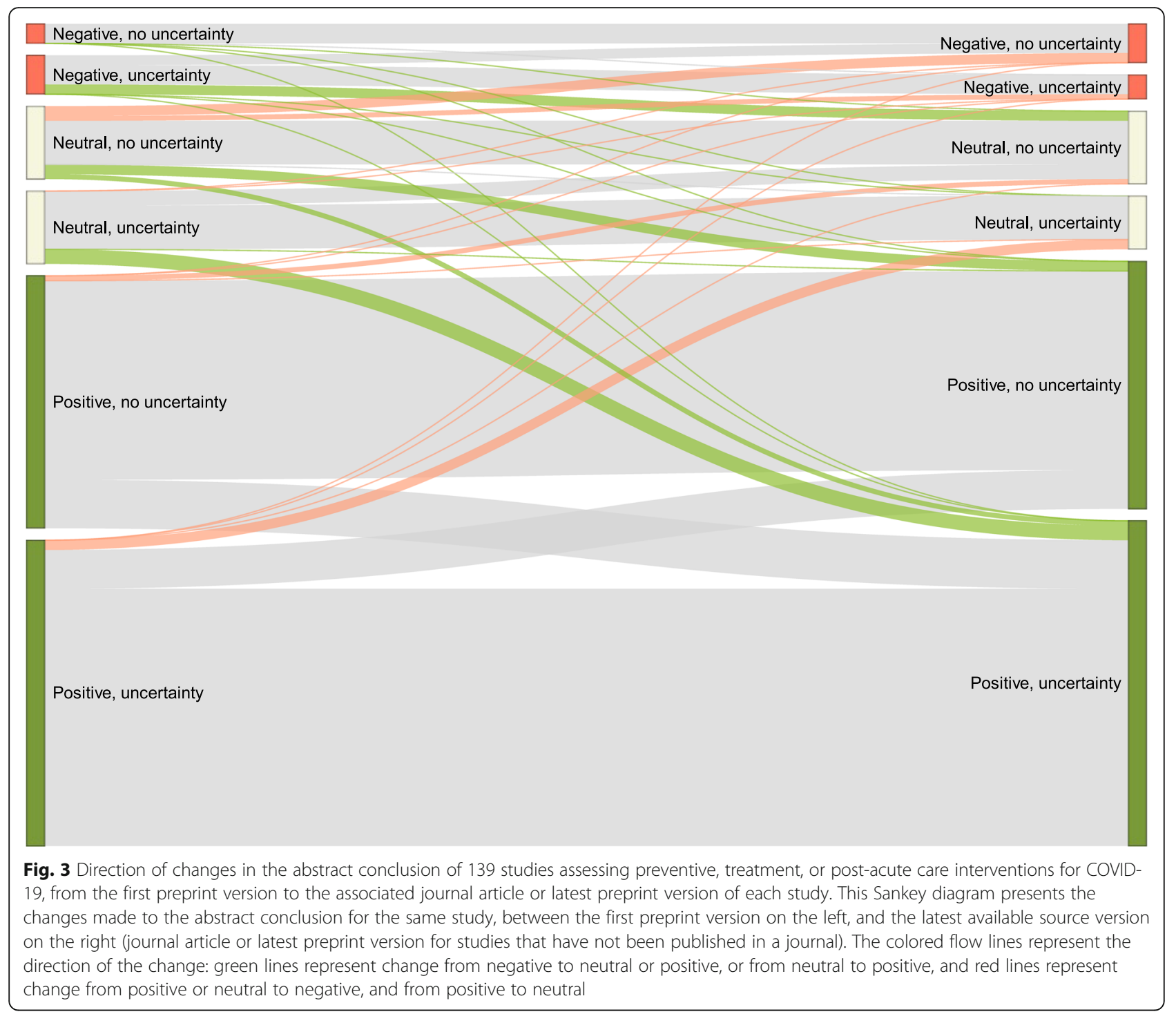

results of systematic reviews and thereby affect decisionmaking. Third, we elicited information about the publication status of preprints by contacting authors. Finally, we designed an algorithm that links preprints to their associated journal articles and flags the appearance of new preprint versions.

Our study has limitations. First, we extracted usage data soon after publication, and a different amount of time had elapsed from the publication of each study to retrieval of usage data. However, preprints tend to receive the majority of views near the time of upload on the server [11]. Additionally, we present usage data normalized by the number of days since publication for each study. Second, we retrieved usage data from the Dimensions database. Had we used a different source, we may have obtained different figures, owing to the different data sources used by each database [15].

\section{Conclusions}

The results of studies assessing the effect of interventions for COVID-19 are often initially disseminated in preprints. The delay in publication of these results in journal articles was $>2$ months, so preprints are a key evidence source for systematic reviews. Studies disseminated as preprints are often reported in multiple preprint versions and in associated journal article, and the evidence reported across these information sources is not stable over time. This situation could affect the results of systematic reviews and thus clinical and policy decision-making. Real-time linkage of all evidence sources for the same study is needed to avoid decisionmaking based on outdated evidence.

\section{Supplementary Information}

Supplementary information accompanies this paper at https://doi.org/10. 1186/s12916-020-01880-8. 
Additional file 1: Methods S1-S3, Tables S1-S2, Figure S1. Methods S1. Search strategy used in the COVID-NMA database. Methods S2 Email template used to contact preprint authors. Methods S3. Data extraction form. Table S1. Sensitivity analysis of change in evidence components using important change in study results. Table S2. Usage data for preprint-journal article pairs. Figure S1. Distribution of usage data in preprint-journal article pairs.

\section{Abbreviations}

COVID-19: Coronavirus disease 2019; RCT: Randomized controlled trial; NMA: Network meta-analysis; PDF: Portable document format; API: Application programming interface; IQR: Interquartile range

\section{Acknowledgements}

The authors thank Laura Smales for editing the manuscript, the study authors who responded to the survey, and the doctoral network of the École des Hautes Études en Santé Publique (EHESP). Theodora Oikonomidi received a fellowship from the doctoral network of the EHESP (Rennes, France). We thank Brandon Stell for providing access to the PubPeer API and Digital Science for providing access to the Dimensions API.

\section{Authors' contribution}

$\mathrm{TO}, \mathrm{PR}$, and IB designed the study and interpreted the results. TO also extracted and analyzed data and drafted the initial manuscript. OP extracted data. GC extracted data and interpreted results. IB is the guarantor of this work. All authors revised and contributed to the draft paper. The corresponding author attests that all listed authors meet authorship criteria and that no others meeting the criteria have been omitted. All authors had full access to all of the data and can take responsibility for the integrity of the data and the accuracy of the data analysis. All authors read and approved the final manuscript.

\section{Funding}

No specific funding has been received for this research. The COVID-NMA project is funded by the Agence Nationale de la Recherche (ANR) and the WHO. Theodora Oikonomidi received a fellowship from the doctoral network of the École des Hautes Études en Santé Publique (EHESP, Rennes, France).

\section{Availability of data and materials}

The datasets generated and/or analyzed during the current study will be made publicly available on https://zenodo.org after this report has been published.

\section{Ethics approval and consent to participate}

Not applicable.

\section{Consent for publication}

Not applicable.

\section{Competing interests}

Theodora Oikonomidi received a fellowship from the doctoral network of the École des Hautes Études en Santé Publique (EHESP, Rennes, France). No other conflicts of interest are reported.

\section{Author details}

'Université de Paris, UMR 1153 CRESS Inserm, 75004 Paris, France. ${ }^{2}$ Clinical Epidemiology Unit, Hôtel-Dieu Hospital, Assistance Publique-Hôpitaux de Paris, (AP-HP), 75004 Paris, France. ${ }^{3}$ Cochrane France, Hôtel-Dieu Hospital, 75004 Paris, France. ${ }^{4}$ University of Toulouse, IRIT UMR 5505 CNRS, 118 route de Narbonne, 31062 Toulouse Cedex 9, France. ${ }^{5}$ Department of Epidemiology, Mailman School of Public Health, Columbia University, New York, NY, USA.

Received: 3 October 2020 Accepted: 4 December 2020

Published online: 17 December 2020

\section{References}

1. Cook DJ, Mulrow CD, Haynes RB. Systematic reviews: synthesis of best evidence for clinical decisions. Ann Intern Med. 1997:126(5):376-80. https:// doi.org/10.7326/0003-4819-126-5-199703010-00006.
2. Horbach SPJM. Pandemic publishing: medical journals drastically speed up their publication process for Covid-19. bioRxiv. Published online April 18, 2020:2020.04.18.045963. https://doi.org/10.1101/2020.04.18.045963.

3. Kwon D. How swamped preprint servers are blocking bad coronavirus research. Nature. 2020;581(7807):130-1. https://doi.org/10.1038/d41586-02001394-6.

4. Boutron I, Chaimani A, Devane D, et al. Interventions for preventing and treating COVID-19: protocol for a living mapping of research and a living systematic review. Published online April 8. 2020. https://doi.org/10.5281/ zenodo. 3744600

5. Herzog C, Hook D, Konkiel S. Dimensions: bringing down barriers between scientometricians and data. Quant Sci Stud. 2020;1 (1):387-95. https://doi. org/10.1162/qss_a_00020.

6. Adie E, Roe W. Altmetric: enriching scholarly content with article-level discussion and metrics. Learn Publ. 2013;26(1):11-7. https://doi.org/10.1087/ 20130103.

7. PubPeer - Search publications and join the conversation. https://pubpeer. com/static/faq\#7. Accessed 12 July 2020.

8. Higgins JP, Savović J, Page MJ, Elbers RG, Sterne JA. Assessing risk of bias in a randomized trial. Cochrane Handb Syst Rev Interv. Published online. 2019: 205-28. https://doi.org/10.1002/9781119536604.ch8.

9. Sterne JA, Hernán MA, Reeves BC, et al. ROBINS-l: a tool for assessing risk of bias in non-randomised studies of interventions. BMJ. 2016;355:14919. https://www.bmj.com/content/355/bmj.i4919.

10. Li Y, Xie Z, Lin W, et al. An exploratory randomized, controlled study on the efficacy and safety of lopinavir/ritonavir or arbidol treating adult patients hospitalized with mild/moderate COVID-19 (ELACOI). medRxiv. Published online 2020:2020.03.19.20038984. doi:https://doi.org/10.1101/2020.03.19. 20038984.

11. Fraser N, Brierley L, Dey G, Polka JK, Pálfy M, Coates JA. Preprinting a pandemic: the role of preprints in the COVID-19 pandemic. bioRxiv. Published online May 23, 2020:2020.05.22.111294. https://doi.org/10.1101/ 2020.05.22.111294.

12. medRxiv.org - the preprint server for Health Sciences. https://www.medrxiv. org/. Accessed 12 July 2020.

13. Kirkham JJ, Penfold N, Murphy F, et al. A systematic examination of preprint platforms for use in the medical and biomedical sciences setting. bioRxiv. Published online April 28, 2020:2020.04.27.063578. https://doi.org/10.1101/ 2020.04.27.063578.

14. Cabanac G. COVID-19 Preprint Tracker. https://www.rit.fr/ Guillaume. Cabanac/covid19-preprint-tracker. Accessed 4 Dec 2020.

15. Visser M, van Eck NJ, Waltman L. Large-scale comparison of bibliographic data sources: Scopus, Web of Science, Dimensions, Crossref, and Microsoft Academic. ArXiv200510732 Cs. Published online May 21, 2020. http://arxiv. org/abs/2005.10732. Accessed 18 July 2020

\section{Publisher's Note}

Springer Nature remains neutral with regard to jurisdictional claims in published maps and institutional affiliations.

Ready to submit your research? Choose BMC and benefit from:

- fast, convenient online submission

- thorough peer review by experienced researchers in your field

- rapid publication on acceptance

- support for research data, including large and complex data types

- gold Open Access which fosters wider collaboration and increased citations

- maximum visibility for your research: over $100 \mathrm{M}$ website views per year

At $B M C$, research is always in progress.

Learn more biomedcentral.com/submissions 